\title{
Galenus-von-Pergamon-Preis 2009 Begehrte Auszeichnung für innovative Arzneimittel
}

\section{Elf pharmakologische Innovationen bewerben sich in diesem Jahr um den von der "Ärzte Zeitung" gestif- teten Galenus-von-Pergamon-Preis. Jedes einzelne Präparat steht für Fortschritt bei der Versorgung von Patienten in Praxis und Klinik und in der Prävention.}

Die Verleihung der national vergebenen Galenus-Preise in Europa hat Tradition: Seit 1970 gibt es diese Auszeichnung für pharmakologische Innovationen und Neuentwicklungen zu Diagnostika. In Deutschland ist dieser nationale Prix Galien der Galenus-vonPergamon-Preis, der 1985 erstmals verliehen wurde und der im Wechsel mit dem Prix Galien International alle zwei Jahre vergeben wird. Der internationale Preis gilt als eine Art Nobelpreis für innovative Arzneimittel.

Der Gewinner des Galenus-von-Pergamon-Preises 2009 wird von einem renommierten wissenschaftlichen Fachkollegium ausgewählt, die Preisverleihung findet im Rahmen einer festlichen Galaveranstaltung im Oktober in Berlin statt.

Quelle: Ärzte Zeitung

\section{Die Bewerber 2009}

Exjade $^{\circledast}$ (Deferasirox)

oraler Chelator gegen Eisenüberladung

Ferinject $^{\circledR}$ (Eisencarboxymaltose)

füllt Eisenspeicher rasch auf

Gardasil $^{\oplus}$

erste Vakzine gegen krebsauslösende

Papillomaviren

Isentress ${ }^{\circledR}$ (Raltegravir)

erster Hemmstoff der Integrase von HIV

Lucentis $^{\circledast}$ (Ranibizumab)

hält die feuchte Makuladegeneration auf

Mezavant ${ }^{\circledR}$ (Mesalazin)

eine Spezialgalenik verlängert die Abgabe des Wirkstoffs im Kolon

$\operatorname{Orfadin}^{\circledast}$ (Nitisinon)

verhindert die Anhäufung toxischer

Metabolite bei angeborener Tyrosinämie

Privigen $^{\circledast}(\mathrm{IVlg}, \lg 10 \%)$

einziges polyvalentes Human-Globulin zur intravenösen Anwendung

Procoralan ${ }^{\oplus}$ (Ivabradin)

Herzfrequenzsenker, der die Reizleitung im Myokard unbehelligt lässt

Targin $^{\circledR}$ (Oxycodon/Naloxon)

das Analgetikum, das die Opioid-induzierte Obstipation verhindert

Tasigna ${ }^{\circledR}$ (Nilotinib)

der Tyrosinkinase-Hemmer hemmt selektiv BCR-ABL, die Ursache der CML

\section{Orfadin ${ }^{\circledast}$ (Nitisinon)}

Orfadin ${ }^{\circledast}$ von Swedish Orphan International enthält den Wirkstoff Nitisinon. Das Präparat ist zugelassen zur Therapie bei angeborener Tyrosinämie Typ 1. Es ist als Orphan Drug ausgewiesen. Die Therapie erfolgt in Kombination mit eingeschränkter Aufnahme von Tyrosin und Phenylalanin durch die Nahrung. Durch Hemmung des normalen Abbaus von Tyrosin mit Nitisinon bei Patienten mit Tyrosinämie Typ 1 wird die Anhäufung toxischer Stoffwechselprodukte verhindert, die die Leber und die Nieren schädigen. Das Risiko für ein Leberkarzinom sinkt. Vor der Einführung von Nitisinon waren eine phenylalanin- und tyrosinfreie oder -reduzierte Diät sowie die Lebertransplantation die einzigen Optionen.

\section{Privigen $^{\circledast}$} (polyvalentes Human-Globulin)

Privigen $^{\circledR}$ von CSL Behring mit einem neuartigen polyvalenten Immunglobulin als Wirkstoff ist ein intravenös applizierbares Immunglobulinpräparat (IVlg, Ig zehn Prozent). Die Anwendungsgebiete sind die Substitutionstherapie bei primären und sekundären Immundefekten, die Immunmodulationstherapie bei Immunthrombozytopenie (ITP), das Guillain-Barré-Syndrom (GBS), Morbus Kawasaki oder nach allogenen Knochenmarktransplantationen. Bei primären Immundefekten etwa lässt sich mit dem Präparat die Rate schwerer bakterieller Infektionen auf 0,08 pro Jahr reduzieren. Trotz hoher Konzentration kann es über 24 Monate uneingeschränkt bei Raumtemperatur gelagert werden.

Quelle: Ärzte Zeitung 